\title{
Star forming cores in L 1251: Maps and molecular abundances
}

\author{
S. Nikolicí ${ }^{1,2}$, L. E. B. Johansson ${ }^{1}$, and J. Harju ${ }^{3}$ \\ ${ }^{1}$ Onsala Space Observatory, 43992 Onsala, Sweden \\ 2 Astronomical Observatory, Volgina 7, 11160 Belgrade 74, Serbia, Serbia and Montenegro \\ ${ }^{3}$ Helsinki University Observatory, Tähtitorninmäki, PO Box 14, SF-00014 University of Helsinki, Finland
}

Received 29 April 2003 / Accepted 17 July 2003

\begin{abstract}
We have mapped the dense parts of the cometary-shaped, star-forming dark cloud L 1251 in the rotational lines of $\mathrm{HCN}, \mathrm{HNC}, \mathrm{HCO}^{+}$and $\mathrm{CS}$ at $3 \mathrm{~mm}$, and observed selected positions in $\mathrm{SO}, \mathrm{CH}_{3} \mathrm{CCH}$ and rare isotopomers of the mapped molecules. Using the CS line we detected 15 cores with sizes of $\sim 0.1-0.3 \mathrm{pc}$. New estimates of the fraction of dense gas in the cores yield a revised average SFE of $\sim 10 \%$. Although 3 times lower than the previous estimate, this high SFE still points to externally triggered star formation in the cloud. Around IRAS $22343+7501$, the source proposed to drive a previously detected extended $\mathrm{CO}$ outflow, our data suggest the existence of either a rotating $\mathrm{HCO}^{+}$disk or a dense outflow with a dynamical age of $\sim 2 \times 10^{4}$ years. A stability check seems to rule out the disk interpretation. We suggest that both continuum sources of Beltrán et al. are protostars each driving its own outflow.

Using methyl acetylene as a thermometer we find indications that at lower temperatures the $A$ and $E$ species are defined by different partition functions. A "temperature gradient" was found in the cloud, with the highest temperature detected in the head region. The column density ratios derived from these observations and the previously published $\mathrm{NH}_{3}$ data show in general little variations, but for two exceptional locations. One of these is in the tip of the "head" with high relative $\mathrm{SO}$ and $\mathrm{NH}_{3}$ abundances, and the other is in the "tail" with low $\mathrm{CO}$ and $\mathrm{HCO}^{+}$column densities with respect to $\mathrm{HNC}, \mathrm{HCN}$ and $\mathrm{NH}_{3}$. In the first case the abundance ratios can probably be explained by an advanced stage of chemical evolution assisted by an elevated temperature. The second location is likely to be an example of $\mathrm{CO}$ and $\mathrm{HCO}^{+}$depletion, and the implication is that also $\mathrm{HNC}$ and $\mathrm{HCN}$ belong to the molecules which are more resistant against freezing-out than $\mathrm{CO}$ and $\mathrm{HCO}^{+}$.
\end{abstract}

Key words. ISM: abundances - ISM: clouds - ISM: molecules - ISM: individual objects: L 1251

\section{Introduction}

The overall star forming efficiency (SFE, defined as the ratio $M_{\text {star }} /\left(M_{\text {star }}+M_{\text {gas }}\right)$, where $M_{\text {gas }}$ is the total gas mass of a molecular cloud and $M_{\text {star }}$ is the mass of embedded protostars) for the Galaxy is estimated to be only 2\% (Myers et al. 1986). Similar low SFEs are typical for dark clouds where low - mass stars are born. However, there are clouds with much higherthan-average SFE which indicates that triggered star formation is significant in such cases. For example supernova shock fronts may stimulate the formation of stars through their local effects on density, kinetic temperatures, turbulence, ionization degrees and, as a result, chemical processes.

L 1251 (Lynds 1962) is an example of a dark cloud with an estimated SFE as high as 30\% (Kun \& Prusti 1993; KP). Both location and cloud morphology suggest that external triggering has contributed to the on-going star formation. This cometary - shaped cloud, at a distance of $300 \mathrm{pc}(\mathrm{KP})$, lies on the Eastern boundary of the Cepheus cloud complex, with the "head" turned towards the center of the Cep-Cas

Send offprint requests to: S. Nikolic, e-mail: silvana@oso.chalmers.se
Void (Grenier et al. 1989). At least two supernovas have exploded in this area within the last $\sim 10^{6}$ years, as indicated by the presence of the major radio-continuum loop, Loop III (Berkhuijsen 1971) and a runaway star HD203854, whose space velocity suggests that it might have been a companion of a supernova some $5 \times 10^{5}-10^{6}$ years ago (Kun et al. 2000). The Cep-Cas Void is suggested to be created by a third SN (Grenier et al. 1989). However, the estimated age of $10^{4} \mathrm{yr}$ means that this $\mathrm{SN}$ is much too young to have affected the star forming processes observed now.

Detected $\mathrm{H} \alpha$ stars in the vicinity of the cloud (Kun 1982) and seven embedded YSO (KP) indicate that the cloud is an active low-mass star formation site. Of the YSOs, IRAS 22376+7455 and IRAS 22343+7501, classified as Class I YSOs (Mardones et al. 1997) apparently power two detected CO outflows (Schwartz et al. 1988; Sato \& Fukui 1989; Sato et al. 1994). Herbig-Haro objects (Balázs et al. 1992; Eiroa et al. 1995) and $\mathrm{H}_{2} \mathrm{O}$ masers (Tóth \& Walmsley 1994; Wilking et al. 1994; Xiang \& Turner 1995; Claussen et al. 1996; Tóth \& Kun 1997) are observed in their vicinity.

The overall distribution of molecular gas in L 1251 has been previously studied by Sato et al. (1994) and 


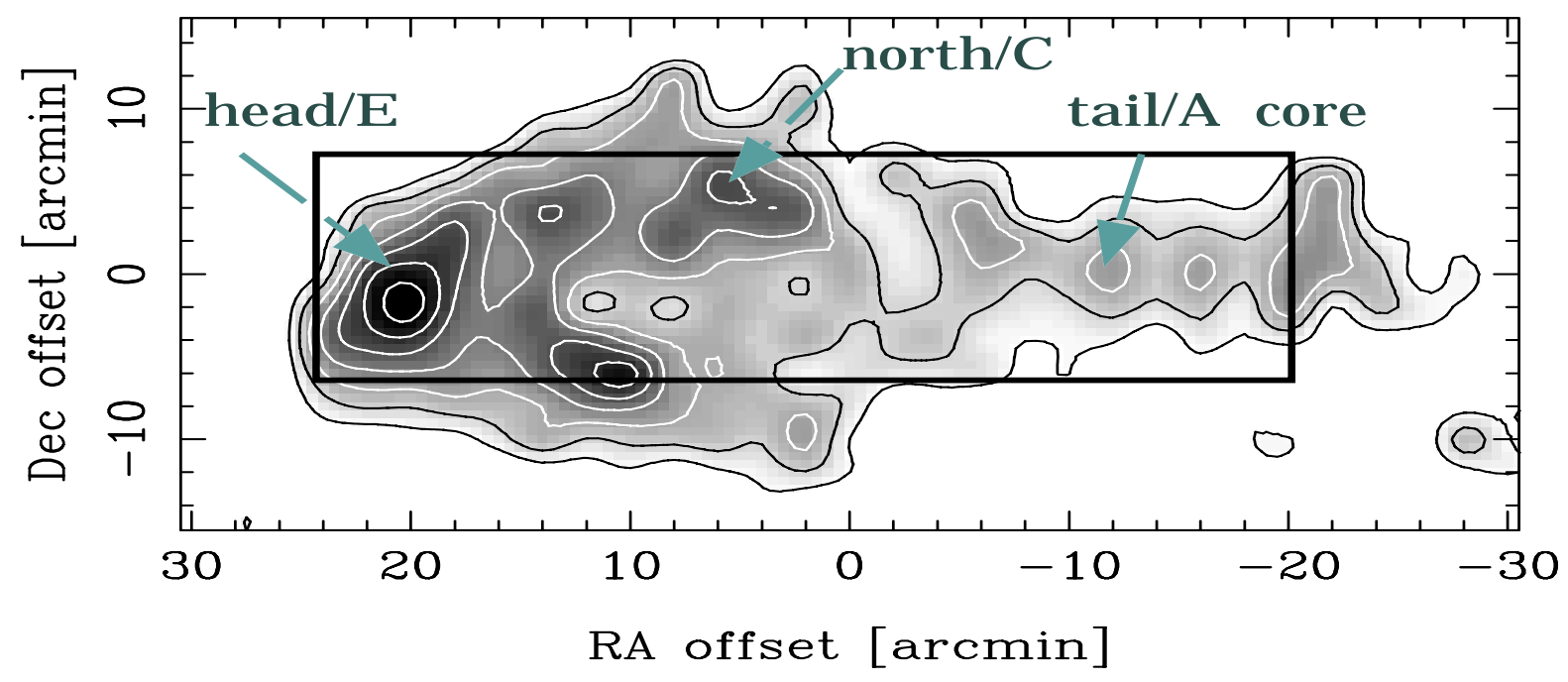

Fig. 1. The ${ }^{13} \mathrm{CO}$ integrated intensity map of $\mathrm{L} 1251$ in the $(-2,-6.5) \mathrm{km} \mathrm{s}^{-1}$ velocity range obtained by Sato et al. (1994). The center position is RA $=22^{\mathrm{h}} 33^{\mathrm{m}}$ Dec $=74^{\circ} 58^{\prime}(1950.0)$. For the designations of the regions indicated, see the Introduction. The bold-line polygon outlines the area presented in Figs. 2-4.

Tóth \& Walmsley (1996; TW). Sato et al. identified five $\mathrm{C}^{18} \mathrm{O}$ cores which they designated as "A" to "E" in increasing RA direction (see Fig. 1). In their ammonia survey, TW discovered three regions of dense gas, "head", "north" and "tail"; the "head" region containing 3 ammonia cores (H 1 to $\mathrm{H} 3$ ) and the "tail" region consisting of 4 cores ( $\mathrm{T} 1$ to $\mathrm{T} 4$ ), whereas in the "north" a single ammonia core was detected $(\mathrm{N}$, see their Fig. 3). The ammonia "head" group of cores corresponds to core "E" of Sato el al., group "north" to core "C" and the "tail" group to core "A".

Apart from $\mathrm{NH}_{3}$, commonly used tracers of dense material are $\mathrm{CS}, \mathrm{HCO}^{+}$, and the isomeric molecules $\mathrm{HCN}$ and $\mathrm{HNC}$, because their rotational transitions near $3 \mathrm{~mm}$ are easy to observe and have critical densities higher that $10^{5} \mathrm{~cm}^{-3}$. In this paper we present maps of these molecules in the densest parts of L 1251, and estimate their column densities along with some other molecular species in selected positions. The cloud contains both protostellar and prestellar condensations and shows clear signs of external influence. The physical conditions are therefore likely to vary across the cloud. The aim of this study was to investigate whether any indications of such variations can be traced in the observed molecular lines, and how these possible changes are reflected in chemical abundances.

\section{Observations}

We used the Onsala Space Observatory's (OSO) 20-m telescope over five observing sessions in 1998, 1999 and 2000 to map the cloud in the HCN (1-0), HNC (1-0), CS (2-1) and $\mathrm{HCO}^{+}(1-0)$ transitions. Selected positions were subsequently observed in ${ }^{13} \mathrm{CO}(1-0), \mathrm{C}^{18} \mathrm{O}(1-0), \mathrm{H}^{13} \mathrm{CN}(1-0), \mathrm{HN}^{13} \mathrm{C}(1-$ $0), \mathrm{C}^{34} \mathrm{~S}(2-1), \mathrm{H}^{13} \mathrm{CO}^{+}(1-0), \mathrm{SO}\left(2_{1}-1_{1}\right)$ and $\mathrm{CH}_{3} \mathrm{CCH}\left(5_{K}-\right.$ $\left.4_{K}\right)$. The receiver was a SIS mixer with a typical $T_{\text {rec }}=100 \mathrm{~K}$ (SSB) in the frequency range used. We used a 1600-channel correlator with $20 \mathrm{MHz}$ bandwidth (i.e. a velocity resolution of $0.04 \mathrm{~km} \mathrm{~s}^{-1}$ at $90 \mathrm{GHz}$ ). The HPBW of the telescope at $90 \mathrm{GHz}$ is $45^{\prime \prime}$ and the main beam efficiency is 0.6 . The pointing was checked by observing several $\mathrm{SiO}$ maser sources and we estimate the pointing uncertainty to be about $3^{\prime \prime} \mathrm{rms}$ in Az and El. The observations were made either in the frequency (main isotopes) or the dual beam switching mode (rarer isotopes). The chopper-wheel method was used for the calibration, and the intensity scale is given in terms of $T_{\mathrm{A}}^{*}$. We used mostly a grid point spacing of $30^{\prime \prime}$, however, occasionally a $60^{\prime \prime}$ step was used. The data were reduced using the $\mathrm{XS}^{1}$ package.

\section{The maps}

Our HCN and $\mathrm{HNC}(1-0)$ integrated intensity maps are shown in Fig. 2, and the corresponding $\mathrm{CS}(2-1)$ and $\mathrm{HCO}^{+}(1-0)$ maps are shown in Figs. 3 and 4, respectively. The area mapped in HNC and HCN covers the two ammonia cores in the "head" designated as "H1" and "H2" by TW, the northern part of L 1251, and the cores "T1", "T2" and "T3" in the tail. All these ammonia cores are associated with newly born stars or protostars. The maps in $\mathrm{CS}$ and $\mathrm{HCO}^{+}$include also the starless core "H3" on the western side of the head core group. Also indicated in these figures are the locations of YSOs and T Tau stars probably associated with the cloud. Labeling of these sources in the upper panel of Fig. 2 follows that of Table 3 of KP.

A comparison between our maps and the $\mathrm{NH}_{3}$ map of TW reveals two major differences: i) in $\mathrm{NH}_{3}$ the head and tail regions have similar integrated intensities whereas in $\mathrm{HCN}$, $\mathrm{HNC}, \mathrm{CS}$ and $\mathrm{HCO}^{+}$the head region is clearly brighter. For the latter molecules the higher integrated intensities in the head are due to a larger number of velocity components in the line of sight (see Sect. 3.2); ii) the core around IRAS 22343+7501 (N1a) is not visible in $\mathrm{NH}_{3}$. Ammonia peaks further up in the north near the T Tau star \#9.

\footnotetext{
1 The program is developed by P. Bergman, OSO.
} 

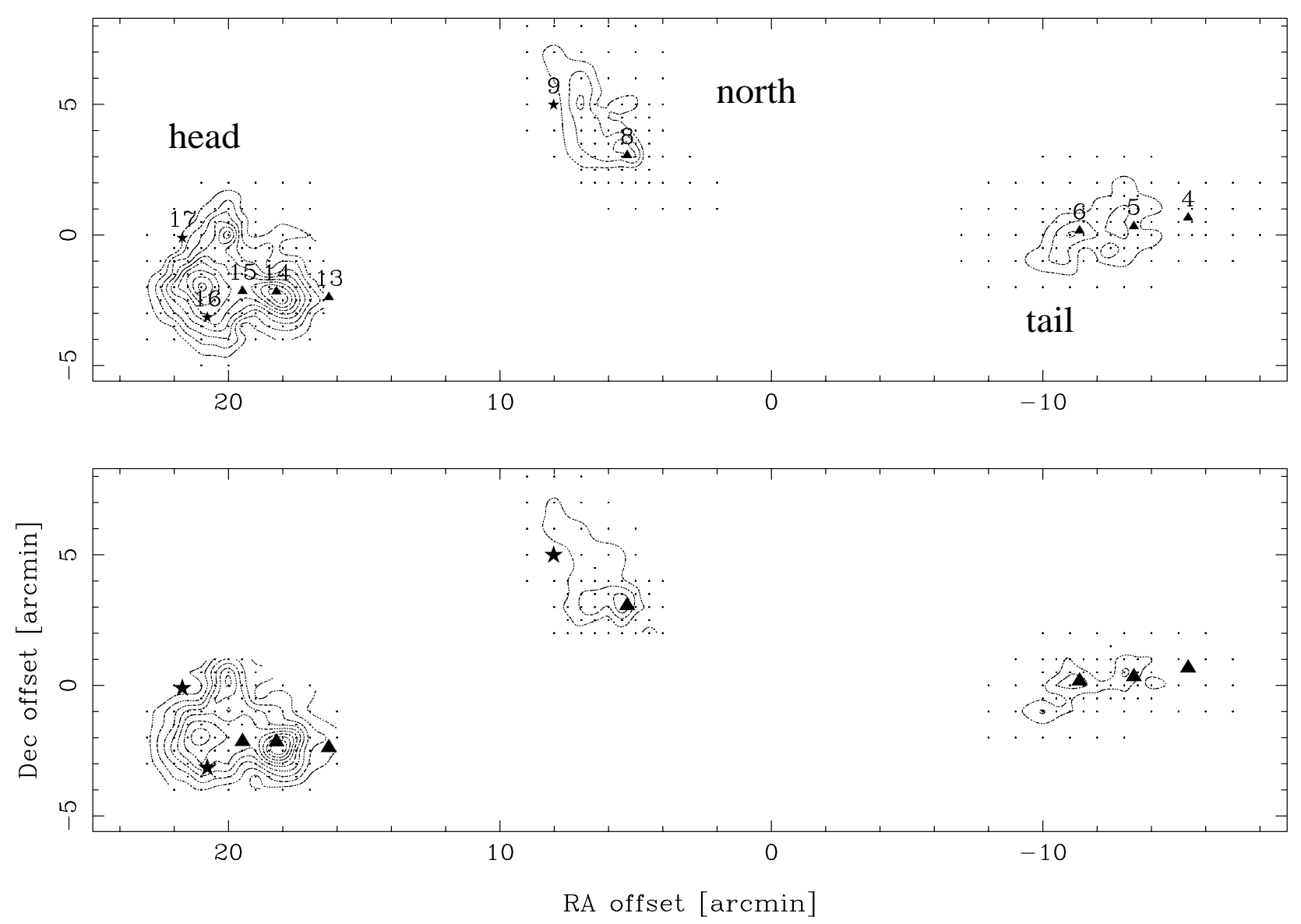

Fig. 2. HNC (upper panel) and HCN (lower panel) integrated intensity maps of L 1251 in the $(-2,-6.5) \mathrm{km} \mathrm{s}^{-1}$ velocity range (for HCN the velocity range of the main $2-1$ component). The center position is the same as in Fig. 1 and the observed positions are indicated by dots. The intensity scale is in $T_{\mathrm{A}}^{*} \mathrm{~d} v\left[\mathrm{~K} \mathrm{~km} \mathrm{~s}^{-1}\right]$ and contours start from 0.9 and increase by 0.45 increments to 4.5 for HNC and from 0.8 with a 0.4 increment to $4.0 \mathrm{~K} \mathrm{~km} \mathrm{~s}^{-1}$ for HCN. The stars denote the FIR point sources classified as T Tau stars, and the triangles those classified as embedded YSOs (from Kun \& Prusti 1993).

\subsection{Identification of cores}

The maps show local maxima which roughly correspond to the ammonia cores detected by TW. Because of the denser sampling and the higher spectral resolution available in the present study we see, however, more structure than discernible in the previous $\mathrm{NH}_{3}$ maps. Using the spatial-velocity information available, we have identified altogether 15 cores in the mapped region, most of which can be seen in all four lines. Some of the $\mathrm{NH}_{3}$ cores of TW divide in our maps into two components. Following the nomenclature of TW we label the five cores studied in detail as H1a, H2a, H2b, N1a and T1a, where "a" and "b" indicate a presence of a secondary peak or a separate velocity component not resolved in the previous $\mathrm{NH}_{3}$ observations.

Table 1 lists the identified cores in order of decreasing RA offset (i.e., in head-to-tail direction). The columns are: (1) core identification number; $(2)$ line center velocity; $(3,4)$ core center in the RA and Dec offsets with respect to the center of the map; (5) full width at half intensity of the integrated emission corrected for the beam size; (6) the full halfwidth of the global line profile of the core; (7) virial mass; (8) mass calculated from $\mathrm{C}^{18} \mathrm{O}$ and (9) association with YSOs or T Tau stars and core designation following TW. The core size, $D$, is estimated from the extent of the half power intensity contour deconvolved with the beam assuming Gaussian shapes for the beam as well as for the source. In most cases cores are elliptical, and we use the geometrical mean of the major and minor axes to define the size. Virial masses are derived using the formula $M_{\text {vir }}=150 D \Delta v^{2}$ (see Johansson et al. 1998). For 5 cores independent mass estimates are derived from the $\mathrm{C}^{18} \mathrm{O}$ observations as $M=1.456 \times 10^{-13} N_{\mathrm{C}^{18} \mathrm{O}}\left(D_{\mathrm{s}}^{2}+D_{\mathrm{b}}^{2}\right)$, where $D_{\mathrm{s}}$ and $D_{\mathrm{b}}$ are source diameter and beam size at the corresponding frequency, respectively (see Nikolić et al. 2001).

We have calculated standard deviation of the core diameters for all available molecules to be in the range $\sim 0.1$ to $\sim 0.3 \mathrm{pc}$. The emission extents of observed molecules agree, in most cases, within $\pm 1 \sigma$ of the arithmetical mean of a core.

The large differences between masses estimated from the virial theorem and the $\mathrm{C}^{18} \mathrm{O}$ data, obvious in three cores, may possibly be linked to the presence of young stellar objects; enhanced turbulence and/or ordered motions like, e.g., outflows could cause the discrepances.

From the $\mathrm{C}^{18} \mathrm{O}$ data, Sato et al. (1994) derived a total gas mass in the head region of $65 M_{\odot}$. Adding up our estimates of core masses in the same region, we arrive at $\sim 75 M_{\odot}$ (using 


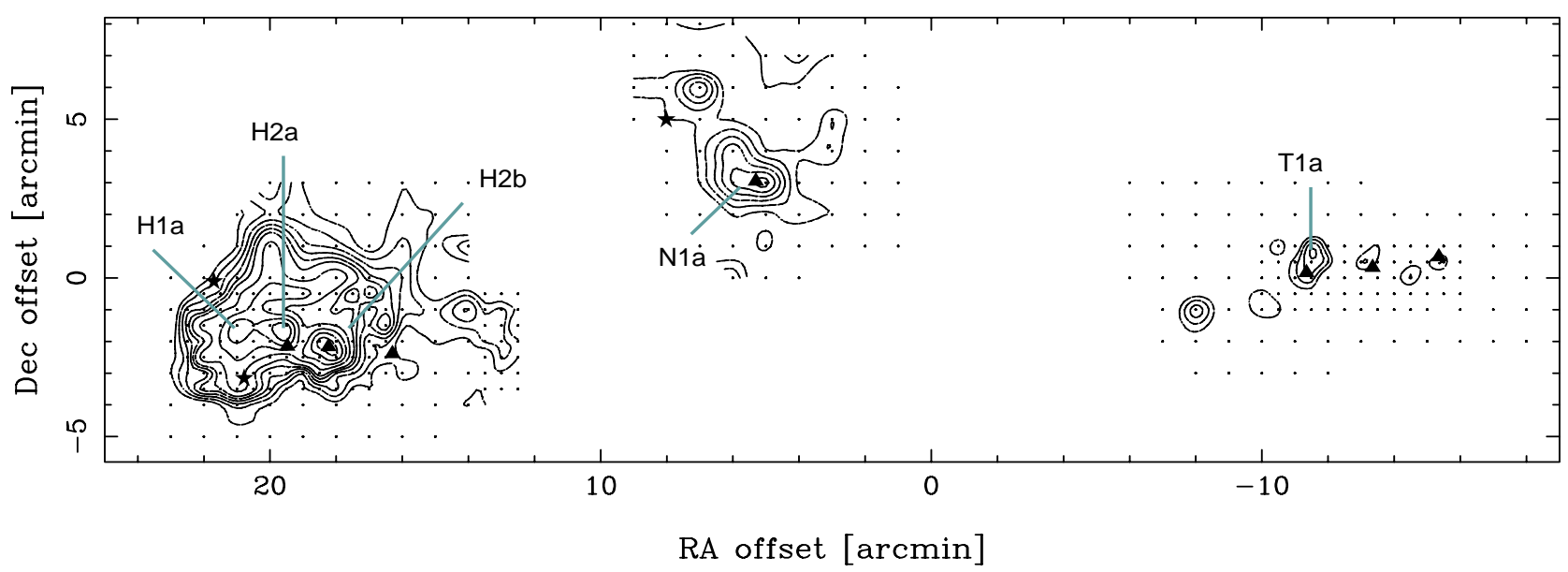

Fig. 3. The CS (2-1) integrated intensity map of L 1251. The velocity range of the emission, the center position, the intensity scale and markers are as in Fig. 1. Contours start from 0.65 to 1.3 by $0.13 \mathrm{~K} \mathrm{~km} \mathrm{~s}^{-1}$ and from 1.3 to 2.34 by $0.26 \mathrm{~K} \mathrm{~km} \mathrm{~s}^{-1}$.

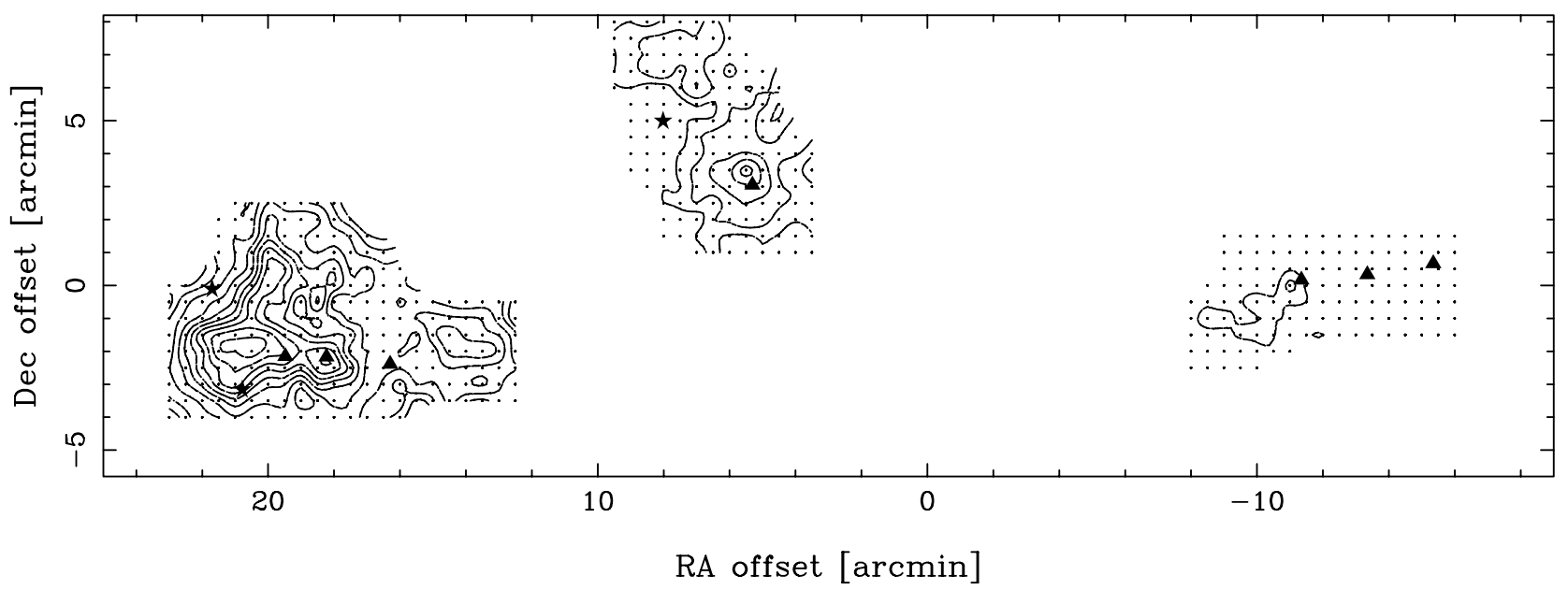

Fig. 4. The $\mathrm{HCO}^{+}(1-0)$ integrated intensity emission map of $\mathrm{L} 1251$ for the $(-2,-8) \mathrm{km} \mathrm{s}^{-1}$ velocity range. The center position, the intensity scale and markers are as in Fig. 1. Contours start from 0.8 and increase by $0.45 \mathrm{~K} \mathrm{~km} \mathrm{~s}^{-1}$.

$M_{\mathrm{CO}}$ where available, otherwise $M_{\mathrm{vir}}$ ), indicating that most of the mass is concentrated in the dense cores. For the northern region of L 1251 Sato et al. (1994) estimate a total gas mass of $56 M_{\odot}$. We derive a mass of $36 M_{\odot}$ for core 7 (i.e. the N1a core) implying that for this region at least $65 \%$ of the total mass is in the form of dense gas.

\subsection{Channel maps and decomposition of the "head" region}

The observed emission in the head covers the range from -6 to $-2 \mathrm{~km} \mathrm{~s}^{-1}$, while the northern area and the tail are seen only in the ranges $(-6,-4)$ and $(-5,-3) \mathrm{km} \mathrm{s}^{-1}$, respectively. This is consistent with the dominant velocity components of the ${ }^{13} \mathrm{CO}$ emission according to Sato et al. (1994).

Figure 3 shows the HNC (1-0) line emission of the head region in four velocity channels. The other observed lines show similar features in the corresponding channel maps. Two velocity components, centered at $\approx-3.5 \mathrm{~km} \mathrm{~s}^{-1}$ and at $\approx-4.5 \mathrm{~km} \mathrm{~s}^{-1}$ are clearly seen in the maps. These two velocity components are also discernible in the $\mathrm{HN}^{13} \mathrm{C}$ spectra of $\mathrm{H} 1 \mathrm{a}, \mathrm{H} 2 \mathrm{a}$ and $\mathrm{H} 2 \mathrm{~b}$.

\subsection{An $\mathrm{HCO}^{+}$"disk" or a dense bipolar outflow?}

Close inspection of the $\mathrm{HCO}^{+}$spectra in the $\mathrm{N} 1 \mathrm{a}$ core area reveals a possible interaction with the $\mathrm{CO}$ outflow (Sato \& Fukui 1989) within $\sim 2^{\prime}$ from the IRAS $22343+7501$ source. At the estimated distance of the cloud this equals $36000 \mathrm{AU}$. In this area the line shapes of $\mathrm{HCO}^{+}$show significant wing emission between -8 and $-2 \mathrm{~km} \mathrm{~s}^{-1}$ as well as selfabsorption features. Figure 6 shows the extents of the blueand red-shifted emission at the most extreme velocities. The structure presented mimics either a rotating $\mathrm{HCO}^{+}$"disk" or a "toroid" (see, e.g., Torrelles et al. 1983) around the protostar, or a dense $\mathrm{HCO}^{+}$outflow. The dashed line in Fig. 6 gives roughly the orientation of the $\mathrm{CO}$ outflow axes. If the structure is considered to be a disk, then the disk radius is $\approx 10000 \mathrm{AU}$. Such large $\mathrm{HCO}^{+}$"disks" around low-mass protostars are not uncommon (see, e.g. Fridlund et al. 2002). On the other side, if we are observing a dense outflow, then the dynamical age is approximately the same for both wings and is equal to $2 \times 10^{4}$ years. 
Table 1. Derived parameters from the CS (2-1) observations. Sizes and masses assume a distance of $300 \mathrm{pc}$.

\begin{tabular}{|c|c|c|c|c|c|c|c|c|}
\hline Core & $\begin{array}{r}v_{\mathrm{lsr}}{ }^{1)} \\
{\left[\mathrm{km} \mathrm{s}^{-1}\right]}\end{array}$ & $\begin{array}{l}\Delta \alpha \\
{\left[{ }^{\prime}\right]}\end{array}$ & $\begin{array}{r}\Delta \delta \\
{\left[{ }^{\prime}\right]}\end{array}$ & $\begin{array}{c}D \\
{[\mathrm{pc}]}\end{array}$ & $\begin{array}{c}\Delta v \\
{\left[\mathrm{~km} \mathrm{~s}^{-1}\right]}\end{array}$ & $\begin{array}{c}M_{\mathrm{vir}}^{2)} \\
{\left[M_{\odot}\right]}\end{array}$ & $\begin{array}{r}M_{\mathrm{CO}}{ }^{3)} \\
{\left[M_{\odot}\right]}\end{array}$ & Comments ${ }^{4)}$ \\
\hline 1 & -4.8 & 21.0 & -1.5 & 0.26 & 1.30 & 66 & 16 & $\mathrm{~T} \mathrm{Tau}\left(\mathrm{H}_{\alpha}\right) \# 16 ; \mathrm{H} 1 \mathrm{a}$ \\
\hline 2 & -4.2 & 20.0 & -0.5 & 0.16 & 0.75 & 14 & & \\
\hline 3 & -3.3 & 19.5 & -1.5 & 0.14 & 0.74 & 11 & 11 & T Tau candidate star \#15; H2a \\
\hline 4 & -4.3 & 18.5 & -2.0 & 0.16 & 1.68 & 70 & 8 & embedded YSO \#14, compact outflow; H2b \\
\hline $5^{a}$ & -3.6 & 14.0 & -1.5 & 0.13 & 1.15 & 26 & & H3 \\
\hline $6^{b}$ & -4.6 & 7.0 & 6.0 & 0.16 & 0.98 & 23 & & T Tau candidate star \#9; N 2 \\
\hline 7 & -5.0 & 5.5 & 3.5 & 0.25 & 1.21 & 55 & 36 & embedded YSO \#8, extended outflow; N1 a \\
\hline $8^{c}$ & -4.4 & -8.0 & -1.0 & 0.13 & 0.66 & 8 & & \\
\hline 9 & -4.4 & -10.0 & -1.0 & 0.13 & 0.76 & 11 & & \\
\hline $10^{d}$ & -4.1 & -10.5 & 1.0 & 0.09 & 0.93 & 12 & & \\
\hline 11 & -4.3 & -11.5 & 0.0 & 0.15 & 0.97 & 18 & 4 & embedded YSO \#6; T1a \\
\hline $12^{d}$ & -4.2 & -12.5 & -0.5 & 0.07 & 0.75 & 6 & & \\
\hline $13^{b}$ & -4.0 & -13.0 & 0.5 & 0.08 & 0.76 & 7 & & embedded YSO \#5; T 2 \\
\hline $14^{d}$ & -4.3 & -14.5 & 0.0 & 0.09 & 1.12 & 14 & & \\
\hline $15^{d}$ & -4.4 & -15.5 & 0.5 & 0.08 & 0.97 & 11 & & embedded YSO \#4; T 3 \\
\hline
\end{tabular}

1 Gaussian fitted line center velocity at the peak position of the core.

$2 M_{\text {vir }}=150 D \Delta v^{2}$ (Johansson et al. 1998).

${ }^{3} M_{\mathrm{CO}}=1.456 \times 10^{-13} N_{\mathrm{C}^{18} \mathrm{O}}\left(D_{\mathrm{s}}^{2}+D_{\mathrm{b}}^{2}\right)$.

4 IRAS point sources and detected outflows that fall within the half intensity contour of the core emission. The positions observed in the rarer isotopomers are also indicated.

${ }^{a}$ Surveyed only in $\mathrm{CS}$ and $\mathrm{HCO}^{+} .{ }^{b}$ Not detected in $\mathrm{HCO}^{+} .{ }^{c}$ Not detected in $\mathrm{HCN} .{ }^{d}$ Detected only in CS.

Table 2. Total column densities derived using the LTE assumption (see the text). For the three cores in the "head" appropriate velocity components were used (see Table 1). The formal errors are 10-15\%, which include intensity calibration $(\sim 10 \%)$ and spectral noise.

\begin{tabular}{lcccrrr}
\hline \hline Molecule & {$\left[\mathrm{cm}^{-2}\right]$} & $\mathrm{H} 1 \mathrm{a}$ & $\mathrm{H} 2 \mathrm{a}$ & $\mathrm{H} 2 \mathrm{~b}$ & N1a & $\mathrm{T} 1 \mathrm{a}$ \\
\hline${ }^{13} \mathrm{CO}$ & {$\left[10^{15}\right]$} & 6.1 & 5.9 & 7.5 & 13.9 & 7.9 \\
$\mathrm{C}^{18} \mathrm{O}$ & {$\left[10^{15}\right]$} & 1.2 & 1.9 & 1.6 & 2.7 & 1.1 \\
$\mathrm{C}^{34} \mathrm{~S}$ & {$\left[10^{11}\right]$} & 4.3 & 7.7 & 11.5 & 14.0 & 11.2 \\
$\mathrm{H}^{13} \mathrm{CN}$ & {$\left[10^{11}\right]$} & 2.5 & 5.4 & 3.7 & 2.1 & 6.6 \\
$\mathrm{HN}^{13} \mathrm{C}$ & {$\left[10^{11}\right]$} & 4.4 & 7.6 & 6.3 & 5.5 & 13.8 \\
$\mathrm{H}^{13} \mathrm{CO}^{+}$ & {$\left[10^{11}\right]$} & 4.0 & 5.8 & 3.7 & 8.6 & 4.6 \\
$\mathrm{SO}$ & {$\left[10^{13}\right]$} & 1.8 & 1.0 & 1.0 & 0.7 & 1.0 \\
$\mathrm{NH}_{3}{ }^{a}$ & {$\left[10^{14}\right]$} & 18 & - & 9.9 & - & 22 \\
\hline
\end{tabular}

a From TW; velocity components of the cores in the head are not resolved.

\section{Column densities and relative abundances}

Table 2 gives column densities, derived assuming LTE conditions and optically thin emission, towards selected positions. In addition, it was assumed that the excitation temperature, $T_{\text {ex }}$, is $10 \mathrm{~K}$ for ${ }^{13} \mathrm{CO}$ and $\mathrm{C}^{18} \mathrm{O}$, and $6 \mathrm{~K}$ for the other species which are likely to be subthermally excited (see, e.g., Caselli et al. 2002). The column densities of $C^{34} S$ and $H N^{13} C$ show the largest variations across the cloud. The $\mathrm{C}^{34} \mathrm{~S}$ column density has a minimum towards $\mathrm{H} 1 \mathrm{a}$ in the head, whereas $\mathrm{HN}^{13} \mathrm{C}$ peaks towards T1a in the tail. The column densities of the other molecules change less than by a factor of three. A comparison between different molecules brings forth some pairs with large variations in the column density ratios, and others with minor changes. For example, $\mathrm{H}^{13} \mathrm{CO}^{+} / \mathrm{C}^{18} \mathrm{O}$ and $\mathrm{HN}^{13} \mathrm{C} / \mathrm{H}^{13} \mathrm{CN}$ are roughly constant $\left(\sim 3 \times 10^{-4}\right.$ and $\sim 2$, respectively $), \mathrm{C}^{34} \mathrm{~S} / \mathrm{SO}$ has by far the lowest value towards $\mathrm{H} 1 \mathrm{a}$, and $\mathrm{HN}^{13} \mathrm{C} / \mathrm{C}^{18} \mathrm{O}$ is clearly largest towards T1a.

We have estimated also the $\mathrm{H}_{2}$ column densities, $N\left(\mathrm{H}_{2}\right)$, with the aid of $\mathrm{C}^{18} \mathrm{O}$ and the conversion factor $\left[\mathrm{C}^{18} \mathrm{O}\right] /\left[\mathrm{H}_{2}\right]=$ $1.7 \times 10^{-7}$ determined by Frerking et al. (1982). The $N\left(\mathrm{H}_{2}\right)$ values have been then used to derive the fractional abundances of other observed molecules. In the conversion to the main

Table 3. $\mathrm{H}_{2}$ total column densities (in $\left[10^{21} \mathrm{~cm}^{-2}\right]$ ) obtained from ${ }^{13} \mathrm{CO}$ using a conversion factor of $N\left(\mathrm{H}_{2}\right) / N\left({ }^{13} \mathrm{CO}\right)=4.8 \times$ $10^{5}$ (Dickman \& Clemens 1983) and from $\mathrm{C}^{18} \mathrm{O}$ using the ratio $\left[\mathrm{C}^{18} \mathrm{O}\right] /\left[\mathrm{H}_{2}\right]=1.7 \times 10^{-7}$ (Frerking et al. 1982), and the calculated fractional abundances of the main isotopomers with respect to $\left[\mathrm{H}_{2}\right]$ derived from $\left[\mathrm{C}^{18} \mathrm{O}\right]$.

\begin{tabular}{|c|c|c|c|c|c|c|}
\hline & & H1a & $\mathrm{H} 2 \mathrm{a}$ & $\mathrm{H} 2 \mathrm{~b}$ & N1a & T1a \\
\hline$\left[{ }^{13} \mathrm{CO}\right] \Rightarrow\left[\mathrm{H}_{2}\right]$ & & 2.93 & 2.84 & 3.58 & 6.67 & 3.77 \\
\hline$\left[\mathrm{C}^{18} \mathrm{O}\right] \Rightarrow\left[\mathrm{H}_{2}\right]$ & & 6.76 & 11.41 & 9.65 & 16.06 & 7.12 \\
\hline $\mathrm{CS}$ & {$\left[10^{-9}\right]$} & 1.4 & 1.5 & 2.6 & 1.9 & 3.4 \\
\hline $\mathrm{HCN}$ & {$\left[10^{-9}\right]$} & 2.9 & 3.6 & 2.9 & 1.0 & 7.1 \\
\hline HNC & {$\left[10^{-9}\right]$} & 5.0 & 5.2 & 5.0 & 2.6 & 14.9 \\
\hline $\mathrm{HCO}^{+}$ & {$\left[10^{-9}\right]$} & 4.6 & 3.9 & 3.0 & 4.1 & 4.9 \\
\hline SO & {$\left[10^{-9}\right]$} & 2.8 & 0.7 & 1.0 & 0.5 & 1.3 \\
\hline $\mathrm{NH}_{3}$ & {$\left[10^{-7}\right]$} & 2.6 & - & 1.0 & - & 3.1 \\
\hline
\end{tabular}



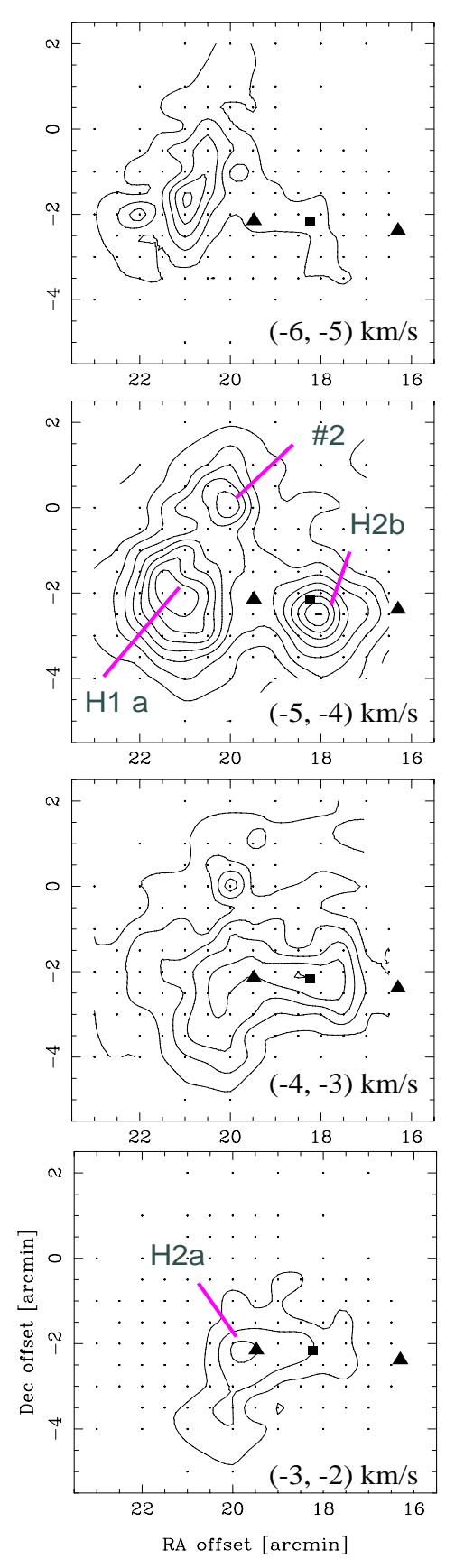

Fig. 5. The HNC channel velocity maps of the "head" region. The observed positions are indicated by dots. The intensity scale is in $T_{\mathrm{A}}^{*} \mathrm{~d} v\left[\mathrm{~K} \mathrm{~km} \mathrm{~s}^{-1}\right]$ and the contours start from 0.15 and are increasing by $0.3 \mathrm{~K} \mathrm{~km} \mathrm{~s}^{-1}$. The velocity ranges are given in the lower right corners. Embedded YSOs are marked with triangles and the YSO suspected to be driving the compact outflow is marked by a rectangle. Additionally, our core designations are given in the second and fourth panel.

isotopomers fractional abundances the following isotopic ratios characteristic of the local ISM have been used: ${ }^{12} \mathrm{C} /{ }^{13} \mathrm{C}=77$ and ${ }^{32} \mathrm{~S} /{ }^{34} \mathrm{~S}=22$ (Wilson \& Rood 1994). The $\mathrm{H}_{2}$ column density estimates and the fractional abundances are given in Table 3. According to this table the position $\mathrm{H} 1 \mathrm{a}$ has the lowest $\mathrm{CS}$ abundance and the largest SO abundance. The fractional HCN and HNC abundances seem to peak towards T1a. N1a, with the highest $\mathrm{H}_{2}$ (in fact $\mathrm{C}^{18} \mathrm{O}$ ) column density, has the

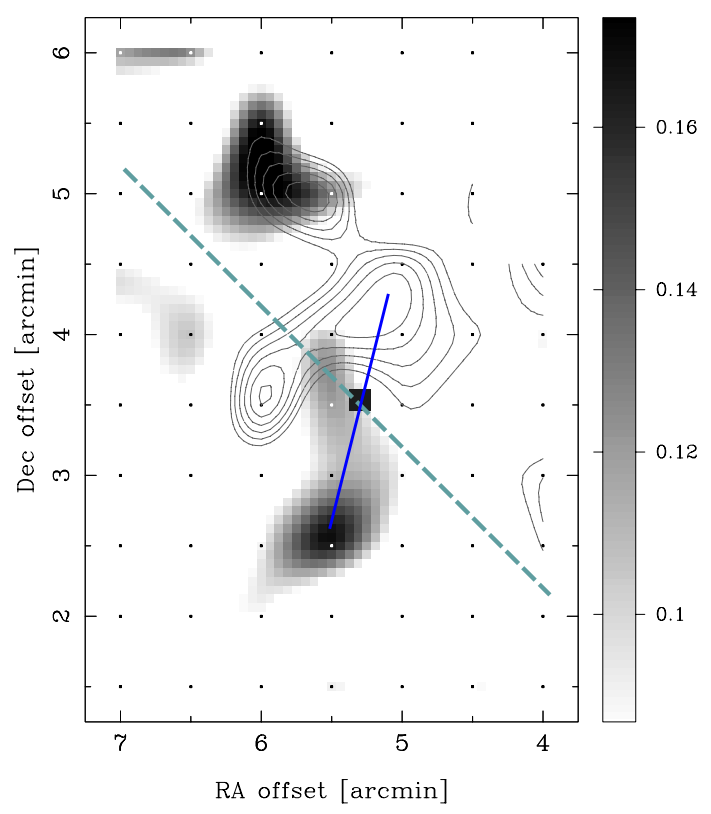

Fig. 6. Integrated intensity map of the blue - (greyscale) and the red-shifted (contours) $\mathrm{HCO}^{+}$emission of the N1a core. The velocity ranges are $(-7,-8)$ and $(-2,-3) \mathrm{km} \mathrm{s}^{-1}$ for the blue and the red wings, respectively. The intensity scale is in $T_{\mathrm{A}}^{*} \mathrm{~d} v\left[\mathrm{~K} \mathrm{~km} \mathrm{~s}^{-1}\right]$ with contours from 0.09 by an increment of $0.01 \mathrm{~K} \mathrm{~km} \mathrm{~s}^{-1}$. The filled square marks the position of IRAS $22343+7501$, the solid line marks the proposed disk and the dashed line shows the orientation of the corresponding CO outflow.

lowest SO abundance. In the other molecules the changes are less marked. It should be noted that the fractional abundances derived here reflect column densities relative to $\mathrm{C}^{18} \mathrm{O}$, and do not represent the true relative abundances with respect to $\mathrm{H}_{2}$ in case the $\mathrm{C}^{18} \mathrm{O} / \mathrm{H}_{2}$ column density ratio changes, e.g., due to CO depletion.

\section{Estimates of temperatures from $\mathrm{CH}_{3} \mathrm{CCH}$}

Symmetric top molecules, like methyl cyanide, $\mathrm{CH}_{3} \mathrm{CN}$, and methyl acetylene, $\mathrm{CH}_{3} \mathrm{CCH}$, make good temperature probes because each $K$-component of a given $J$ rotational transition has a different energy level. Radiative transitions between different $K$-ladders are prohibited by the selection rule $\Delta K=0$; thus the populations of different K-ladders are determined by collisions and depend mostly on the gas kinetic temperature. Also, being relatively close in frequencies, all $K$ components can be observed simultaneously, avoiding calibration problems.

The $J=5-4, K=0,1,2,3$, transitions of methyl acetylene were observed towards the previously selected five cores in L 1251. Figure 7 shows the spectra. To estimate kinetic temperatures we have used the rotational diagram method (see, e.g., Anderson et al. 1999) which assumes LTE conditions and optically thin emission. The derived rotational temperatures are unexpectedly high as are the associated errors. The reason for this can be traced back to the intensity of the $K=0$ relative to the $K=1$ transition; with the exception of the region $\mathrm{H} 1 \mathrm{a}$, the observed ratios all indicate $T_{\text {rot }}>50 \mathrm{~K}$. Such results can be explained in terms of non-LTE excitation or that the 


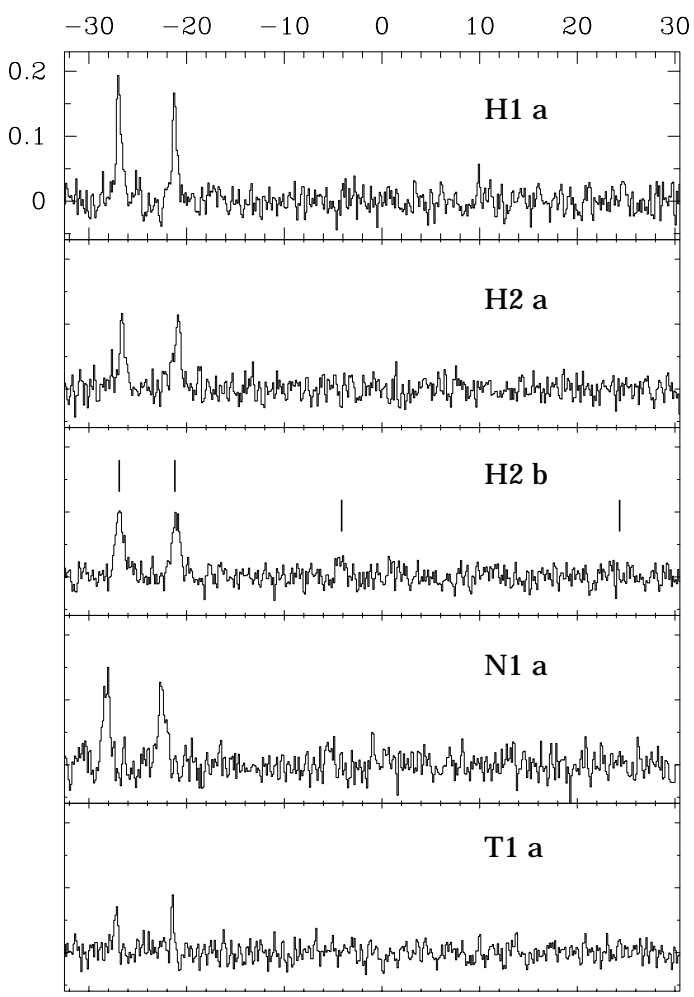

Fig. 7. The observed $\mathrm{CH}_{3} \mathrm{CCH} 5-4$ spectra smoothed to a velocity resolution of $0.12 \mathrm{~km} \mathrm{~s}^{-1}$. Core designation is given in the upper right corner and the observed $K=0,1,2,3$ transitions are marked with solid lines. The intensity scale is $T_{\mathrm{A}}^{*}[\mathrm{~K}]$, and the velocity scale is $v_{\mathrm{LSR}}$ $\left[\mathrm{km} \mathrm{s}^{-1}\right]$.

total abundances of the $A(K=0)$ and $E(K=1)$ species are not equal. Assuming $T_{\text {rot }}=15 \mathrm{~K}$, the $E / A$ population ratio would be about 1.5 based on the $K=0$ and 1 transitions. Askne et al. (1984) find that statistical equilibrium and rotational diagram calculations agree, with the exception of cold regions. Their analysis indicate that the total abundances of the $A$ and $E$ species are equal, however, defined by different partition functions at low kinetic temperatures. For TMC -1 , their observations show an intensity ratio of $\sim 1$ between the $K=0$ and $1, J=5-4$ transitions, while the statistical equilibrium analysis indicates $T_{K} \sim 10 \mathrm{~K}$ and $T_{\text {rot }} \sim 6 \mathrm{~K}$. Observationally, this is very similar to our sample (with the exception of H1a), possibly indicating that this head core is warmer than the rest of the cores observed here.

To proceed we have introduced an uncertainty in the $A$ and $E$ species populations of $30 \%$. The results are given as "case I" in Table 4 and clearly show smaller errors in the derived parameters in spite of larger total errors in the input data, a reflection of the inconsistent intensities of the $K=0$ and 1 transitions. Figure 8 shows graphically this inconsistency common for all our cores with the exception of H1a. In "Case II" we have only used the $K=1$ and 2 transitions (i.e. the $E$-species) to estimate the rotation temperatures. These results are further discussed in Sect. 6.4.
Table 4. The results of the $\mathrm{CH}_{3} \mathrm{CCH}$ rotational diagram method weighted with observational errors plus $\mathrm{E}$, A species uncertainties (case I) and using the $K=1$ and $K=2$ lines only (case II). Ammonia temperatures are from TW.

\begin{tabular}{lrrrrr}
\hline \hline & H1a & H2a & H2b & N1a & T1a \\
\hline case I & & & & & \\
$T_{\text {rot }}[\mathrm{K}]$ & $27 \pm 6$ & $16 \pm 5$ & $18 \pm 2$ & $23 \pm 2$ & $22 \pm 7$ \\
$N_{\text {tot }}\left[10^{12} \mathrm{~cm}^{-2}\right]$ & $31 \pm 8$ & $16 \pm 8$ & $21 \pm 4$ & $27 \pm 5$ & $8 \pm 3$ \\
case II & & & & & \\
$T_{\text {rot }}[\mathrm{K}]$ & $15 \pm 4$ & $8 \pm 2$ & $15 \pm 2$ & $16 \pm 3$ & $10 \pm 5$ \\
$N_{\text {tot }}\left[10^{12} \mathrm{~cm}^{-2}\right]$ & $25 \pm 2$ & $27 \pm 5$ & $23 \pm 1$ & $28 \pm 2$ & $8 \pm 1$ \\
$T_{21}^{\mathrm{NH}}[\mathrm{K}]$ & 10 & 12 & 14 & - & 10 \\
\hline
\end{tabular}

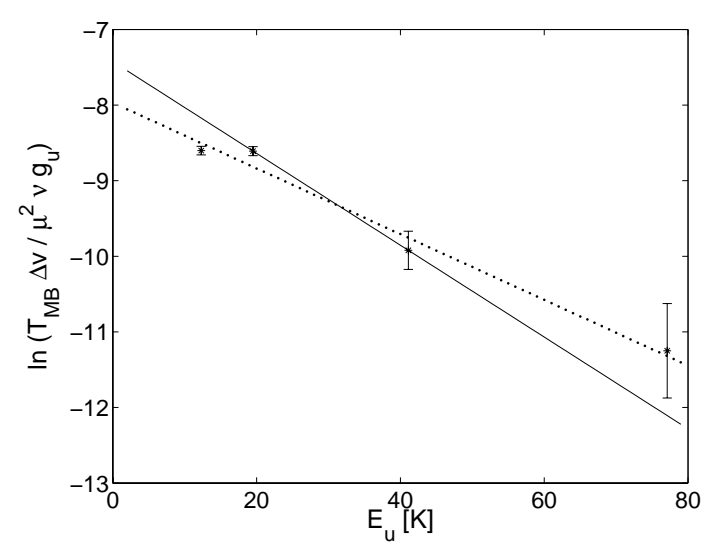

Fig. 8. Rotation diagram of the $\mathrm{CH}_{3} \mathrm{CCH} 5-4$ transition for core N1a. For definition of case I (dotted line) and case II (solid line) see the text and Table 4 . The error bars refer to the observational uncertainties only.

\section{Discussion}

\subsection{Kinematics of the cloud}

Our high spectral resolution observations have revealed two distinct velocity components in the head region, separated by about $1 \mathrm{~km} \mathrm{~s}^{-1}$. The same components are most likely present in other molecules as well. The fact that these were interpreted as velocity gradients by TW is due to their cruder velocity resolution.

Similarly, a superposition of these two components at least partly explains the observed CS line asymmetry, i.e., "the infall profiles" seen by Mardones et al. (1997) towards IRAS $22376+7455$. Yet a possible infall of dense gas cannot be ruled out.

\subsection{YSOs and T Tau stars in dense cores}

Five out of six embedded YSOs, as proposed by KP in the region we have surveyed, are most likely associated with the denser parts of L 1251: FIR sources \#4, \#5 and \#6 in the tail area (associated with the cores 15, 13 and 11 in Table 1, respectively), source \#8, (IRAS 22343+7501) in the northern area with core 7 (N1a) and source \#14 (IRAS 22376+7455) with 
core $4(\mathrm{H} 2 \mathrm{~b})$. Additionally, based on their projection onto dense areas, three $\mathrm{T}$ Tau candidate stars are probably associated with the cloud: sources \#16 and \#17 in the topmost part of the head area and source \#9 in the northern area of the cloud, all coincident with detected $\mathrm{H} \alpha$ emission stars. Sources \#13 and \#15 in the head area are both blended by the strong IR emission from IRAS $22376+7455$, and have ill-defined error ellipses. Since the former source does not seem to be associated with any dense core it may be either only projected on the cloud area or is a faint, low-mass embedded star (Kun, priv. com.). The position of source \#15 correlates well with the center of core 3 (see Fig. 5), where the HCN peak integrated emission is $\sim 2.5 \mathrm{~K} \mathrm{~km} \mathrm{~s}^{-1}$. Yun et al. (1999) derived an $80 \%$ likelihood of tracing an embedded Class 0 YSO if the detected HCN emission is stronger than $3 \mathrm{~K} \mathrm{~km} \mathrm{~s}^{-1}$. Thus, source \#15 may be an embedded Class 0/I YSO.

To derive the SFE of the cores we assume $M_{*} \sim 1 M_{\odot}$ for all the embedded YSOs and TTau stars with unknown masses, i.e., sources \#6, \#15, \#16 and \#17. IRAS 22376+7455 and IRAS $22343+7501$ have estimated masses of $1.78 M_{\odot}$ and $2.35 M_{\odot}$, respectively (Kun 1998). If we use the masses of the cores listed in Table $1\left(M_{\mathrm{CO}}\right)$, SFEs for the H1a, H2a, H2b, N1a and T1a cores are approximately $11 \%, 9 \%, 18 \%, 6 \%$ and $20 \%$, respectively. On average, SFE of the observed cores would be $\sim 13 \%$, almost 3 times lower than the previously estimated SFE for the whole cloud, but still 5-6 times higher than the overall SFE for the Galaxy.

\subsection{Nature of IRAS $22343+7501$}

Based on IRAS and sub-mm continuum observations Mardones et al. (1997) derived the bolometric temperature of IRAS $22343+7501$ to be $\leq 108 \mathrm{~K}$, and classified the source as a Class I YSO. Using Chen et al. (1995) empirical relation between age and bolometric temperature for YSOs with $T_{\text {bol }} \leq 1000 \mathrm{~K}$, we estimate IRAS $22343+7501$ to be $(1.3 \pm$ $0.1) \times 10^{4}$ years old. This age would classify the source as a very young Class I YSO. Sato et al. (1994) derived the dynamical timescale (using an inclination of $45^{\circ}$ ) of the $\mathrm{CO}$ outflow to be $9.2 \times 10^{4}$ and $1.8 \times 10^{5}$ years for the blue- and the red-wing, respectively. Provided that IRAS $22343+7501$ is the $\mathrm{CO}$ outflow driving source, consistent age estimates would require that the flow plane is close to the plane of sky, i.e., tilted by $4-8^{\circ}$. Kun (1998) estimated the mass of a hypothetical central star to be $M_{*}=2.35 M_{\odot}$. Near-infrared images (Rosvick \& Davidge 1995) and recent $3.6 \mathrm{~cm}$ and $6 \mathrm{~cm}$ VLA continuum observations (Grissom Meehan et al. 1998; Beltrán et al. 2001) show that this IRAS source actually consists of several protostellar objects. The near-IR images exhibit a 20-30" large nebulosity corresponding to a maximum size of $9000 \mathrm{AU}$. VLA continuum measurements revealed two sources, separated by $7^{\prime \prime}$, i.e., about 2000 AU. Both continuum sources have spectral indices consistent with thermal emission, and any of them could be the $\mathrm{CO}$ outflow driving source (Beltrán et al. 2001). The proposed $\mathrm{HCO}^{+}$disk would encompass both sources.
Under the assumption that the properties of the $\mathrm{HCO}^{+}$ emission (see Fig. 6) are similar to those in the N1a core, i.e., $I\left(\mathrm{HCO}^{+}\right) / I\left(\mathrm{H}^{13} \mathrm{CO}^{+}\right) \approx 7$, we derive a total mass of this feature as $M=50 \times I_{M B}\left(\mathrm{HCO}^{+}\right) A\left[M_{\odot}\right]$, where $I_{M B}$ is the velocity integrated emission $\left[\mathrm{K} \mathrm{km} \mathrm{s}^{-1}\right]$ and $A$ is the area of the $\mathrm{HCO}^{+}$wing emission $\left[\mathrm{pc}^{2}\right]$. It is further assumed that the isotopic abundance ratio is 77 and that the fractional abundance of $\mathrm{HCO}^{+}$ is $4 \times 10^{-9}$ (see Table 3 ). The total wing emission integrated over the full velocity range and wings areas is $0.1 \mathrm{~K} \mathrm{~km} \mathrm{~s}^{-1}$, yielding $\approx 5 M_{\odot}$ of molecular gas, equally distributed between the approaching and receding emission regions.

Assuming that this emission originates from a disk rotating with a velocity of $\sim 2 \mathrm{~km} \mathrm{~s}^{-1}$ at its outer edge we find the centrifugal force to be $\sim 12$ times larger than the gravitational force of the central star and the "disk" combined. Since the observed radial velocity is only a lower limit to the "rotation velocity", this factor (proportional to $v_{\text {rot }} / M$ ) can most likely be considered as a lower limit, although our derived mass could be underestimated if the $\mathrm{HCO}^{+}$is more saturated than in the surroundings. However, the $\mathrm{H}^{13} \mathrm{CO}^{+}$spectrum toward the IRAS source (N1a core center) shows no signs of wing emission, indicating a saturation level of the main isotope emission similar or lower than assumed. Based on our high ratio of centrifugal to gravitational forces we thus find it unlikely that the observed $\mathrm{HCO}^{+}$wing emission defines a disk.

On the other hand, if the observed structure represents a dense outflow, the derived dynamical age of the flow is $2 \times 10^{4}$ years (not corrected for an unknown inclination), i.e., an order of magnitude less than the $\mathrm{CO}$ outflow. The discrepancy between the dynamical ages for the $\mathrm{CO}$ and the suggested $\mathrm{HCO}^{+}$outflow indicates that they are of different origin. This is further emphasized by the apparently different orientations of the flows in the plane of the sky as well as in the radial direction (in contrast to $\mathrm{CO}$, the red-and blue-emission regions of $\mathrm{HCO}^{+}$partly overlap). As noted earlier, we can probably treat the derived mass of $\sim 5 \mathrm{M}_{\odot}$ in the $\mathrm{HCO}^{+}$wings as an upper limit. We define a lower limit by assuming optically thin $\mathrm{HCO}^{+}$wing emission and arrive at $\sim 0.5 \mathrm{M}_{\odot}$. Using this range of masses, the released kinetic energy of the $\mathrm{HCO}^{+}$flow is estimated to be $4-40 \times 10^{36} \mathrm{~J}$ and mechanical luminosity to be $1-10 \times 10^{25} \mathrm{~J} / \mathrm{s}$ i.e., $0.03-0.3 L_{\odot}$; the lower limit comparable to the mechanical luminosity of the $\mathrm{CO}$ outflow although the $\mathrm{CO}$ outflow is spread over an area of the order of a magnitude larger. In this picture of two outflows traced by the $\mathrm{CO}$ and the $\mathrm{HCO}^{+}$emission, both continuum sources of Beltrán et al. (2001) could be protostars each driving its own outflow.

\section{4. $\mathrm{HCN} / \mathrm{HNC}$ ratios and kinetic temperatures}

According to gas - phase chemical models the main route for production of $\mathrm{HCN}$ and $\mathrm{HNC}$ is by dissociative recombination with electrons (e.g. Hirota et al. 1998):

$\mathrm{HCNH}^{+}+\mathrm{e}^{-} \rightarrow \mathrm{HNC}+\mathrm{H}, \mathrm{HCN}+\mathrm{H}$.

Based on their observations of starless and star forming cores, Hirota et al. (1998) derived a branching ratio of the reaction of 0.4 for the HNC production. The branching production ratio of $\mathrm{HCN}, \alpha$, is defined as $[\mathrm{HCN}] /[\mathrm{HNC}]=\alpha / 1-\alpha$, if 
$T_{\text {kin }} \ll T_{\mathrm{c}}$, where $T_{\mathrm{c}} \approx 24 \mathrm{~K}$ is the threshold temperature above which neutral-neutral reactions dominate the $\mathrm{HCN} / \mathrm{HNC}$ ratio. We have estimated $\alpha$ in the directions of the sample cores. In the head part of L 1251 both total emission and the previously defined core emission is considered. All cores i.e., H1a, H2a, $\mathrm{H} 2 \mathrm{~b}, \mathrm{~N} 1 \mathrm{a}$ and $\mathrm{T} 1 \mathrm{a}$ have a branching production ratio of $\mathrm{HCN}$ in the range $\alpha=0.2-0.4$. Similar values in the $\mathrm{H} 2 \mathrm{a} / \mathrm{b}$ cores are observed also when the total velocity ranges of the HCN and HNC emission are considered. However, towards the H1a core the branching ratio for the $\mathrm{HCN}$ production of the total emission is as high as $\alpha=0.8 \pm 0.1$. The latter ratio is probably a reflection of more energetically favorable paths for destruction of $\mathrm{HNC}$ once the temperature of the gas exceeds the critical temperature in the region. Thus, this ratio indicates $T_{\text {kin }} \geq 24 \mathrm{~K}$, while the rest of the cores are colder. This pattern is favored by the temperatures derived from our $\mathrm{CH}_{3} \mathrm{CCH}$ observations (Table 4), although one should bear in mind the relatively large errors. However, as pointed out in Sect. 4 there are indications that the partition function of the $A$ and $E$ species deviate at low temperatures and, in our simple analysis, overestimate the temperatures. This effect would then apply to all our cores except towards H1a.

In conclusion, our $\mathrm{HCN} / \mathrm{HNC}$ and $\mathrm{CH}_{3} \mathrm{CCH}$ results favor a kinetic temperature in excess of $\sim 25 \mathrm{~K}$ towards $\mathrm{H} 1 \mathrm{a}$ and less or significantly less than $\sim 20 \mathrm{~K}$ for the rest of the cores. This temperature increase of the dense gas is likely caused by shocks originating from outflows and/or radiation from the embedded stars. We searched the 2MASS All Sky Catalog and in the head region of $\mathrm{L} 1251$, clustered around the two $\mathrm{H}_{\alpha}$ stars and the two embedded YSOs (\#14 and \#15), we found 23 point sources. This suggests that the head region of L 1251 is much more productive than the northern core ( 8 point sources), and the tail cores (6 point sources). Regarding the possibility of external heating, the newly born pre-main sequence stars observed by $\mathrm{KP}$ could, in principle, heat the outer parts of the cloud to 20 $25 \mathrm{~K}$. However, there the gas density is too low to excite the molecular transitions observed by us. Another possibility, as suggested in the Introduction, is that the cloud has encountered at least one external shock; however such shocks have cooling times in a dense gas of the order of years (see, e.g., Smith \& Rosen 2003).

\subsection{Star formation and "Early-" and "Late-time" molecules}

Based on the production pathways via ion-molecule or neutralneutral reactions, and their dependence on neutral carbon (C I), some molecules are classified either as "early-time" $\left(10^{4}-\right.$ $10^{6}$ years after the onset of chemistry) or "late-time" species (maximum abundances reached at steady state, after about $10^{6}-$ $10^{8}$ years of chemical evolution; e.g. Herbst \& Leung 1989). Of the molecules discussed here $\mathrm{HCO}^{+}, \mathrm{SO}$ and $\mathrm{NH}_{3}$ are usually considered as late-time molecules; $\mathrm{HCO}^{+}$because it is formed from $\mathrm{CO}$, and the latter two because their formation mechanisms involve relatively slow neutral-neutral reactions. SO is furthermore destroyed primarily by $\mathrm{CI}$ which at later stages is locked up in CO (e.g. Nilsson et al. 2000). CS forms early, but its abundance remains roughly constant because of recycling via $\mathrm{HCS}^{+}$(Nejad \& Wagenblast 1999). The situation of $\mathrm{HCN}$ and $\mathrm{HNC}$ is less clear in this picture. The precursor ion, $\mathrm{HCNH}^{+}$, is produced mainly via a reaction between $\mathrm{NH}_{3}$ and the $\mathrm{C}^{+}$ion, thus involving both a typical "late-time" molecule and an ion characteristic of young chemistry.

In the recent models of Rawlings et al. (2002) it is shown that in an "H-rich" environment the nitrogen chemistry is initiated at an early stage, and because of the less effective destruction of $\mathrm{He}^{+}$(due to a low $\mathrm{CO}$ abundance), $\mathrm{NH}_{3}$ forms quickly thereafter. Under these circumstances also $\mathrm{HNC}$ is produced at early times via $\mathrm{C}+\mathrm{NH}_{2}$. $\mathrm{NH}_{2}$ forms from a dissociative recombination of $\mathrm{NH}_{3}^{+}$, which is one of the precursors of ammonia. In the "H-poor" models of Rawlings et al., on the other hand, the formation of $\mathrm{HCN}$ is efficient via $\mathrm{N}+\mathrm{CH}_{2}$ or $\mathrm{N}+\mathrm{CH}_{3}$ at early times. From these results one can expect that at early stages of chemical evolution the HCN/HNC abundance ratio depends strongly on the initial $\mathrm{H}$ content.

The division of molecules into "early-time" and "latetime" species is valid eventually only until a protostar is formed in a core, as this may change the physical conditions and consequently the chemical composition of the ambient cloud via heating of dust grains, enhanced turbulence and radiation field (see, e.g. Nejad et al. 1990). As a consequence of intensified desorption and ionization, we are then able to observe characteristically "young" chemistry.

$\mathrm{X}$ - ray surveys of star forming regions showed that Class I-III YSOs have significant $\mathrm{X}$-ray emission (e.g., Getman et al. 2002; Casanova et al. 1995). The heating effect of this emission is very localized (e.g., Lepp \& McCray 1983), but the $\mathrm{X}$-ray induced ionization affects the whole core/cloud. Casanova et al. (1995) derived X-ray induced ionization rate throughout the $\rho$ Oph cloud core to be comparable with the usually assumed cosmic rays ionization rate of $\sim 10^{-17} \mathrm{~s}^{-1}$. A paradoxical situation may occur in the sense that a dynamically older core, with a central Class 0 embedded YSO is chemically younger than a pre-protostellar core (Kontinen et al. 2000).

All the dense cores studied here are associated with protostars or newly born stars: the presence of T Tau stars in the vicinity of $\mathrm{H} 1$ a would indicate a dynamical age $\geq 10^{6}-10^{7}$ years for this core, while the embedded YSOs (\#6, \#8, \#14 and \#15) in the remaining 4 cores point to ages of $10^{4}-10^{5}$ years, with the tail core being the youngest. The fact that H1a is located near the compression front corroborates the notion that the core is the most evolved among the L 1251 cores.

The fractional abundances given in Table 3 and the column density ratios given in Table 5 show some differences but lack clear trends with respect to the adopted dynamical ages of the cores. The large $\mathrm{SO} / \mathrm{CS}$ and $\mathrm{NH}_{3} / \mathrm{CS}$ column density ratios in $\mathrm{H} 1 \mathrm{a}$ in the head conform with the idea that this core has reached an advanced stage of evolution. However, as discussed above one would possibly expect the presence of a T Tau star to alter the chemistry towards "younger" stages. This suggests that the influence of this T Tau star is hardly significant, provided that present chemical networks of dense and cold clouds predict the evolution of molecular abundances in a rather accurate way. On the other hand, the relatively low $\mathrm{CO}$ and $\mathrm{HCO}^{+}$column densities in T1a with respect to $\mathrm{NH}_{3}$ and $\mathrm{HNC}$ (see Table 3) can 
Table 5. Ratios of column densities, based on Table. 3. Relative errors of the ratios are in the range of $5-15 \%$, for $\mathrm{SO}, \mathrm{CS}, \mathrm{HCO}^{+}$and $\mathrm{HNC}$. For ratios that include ammonia, errors are in the range $20-25 \%$.

\begin{tabular}{lccrrrrr}
\hline \hline & $\frac{[\mathrm{SO}]}{[\mathrm{CS}]}$ & $\frac{\left[\mathrm{HCO}^{+}\right]}{[\mathrm{CS}]}$ & $\frac{\left[\mathrm{NH}_{3}\right]}{[\mathrm{CS}]}$ & $\frac{[\mathrm{SO}]}{[\mathrm{HNC}]}$ & $\frac{\left[\mathrm{HCO}^{+}\right]}{[\mathrm{HNC}]}$ & $\frac{\left[\mathrm{NH}_{3}\right]}{[\mathrm{HNC}]}$ \\
\hline H1a & 2.0 & 3.2 & 186 & 0.6 & 0.9 & 53 \\
H2a & 0.5 & 2.6 & - & 0.1 & 0.8 & - \\
H2b & 0.4 & 1.1 & 39 & 0.2 & 0.6 & 20 \\
N1a & 0.2 & 2.1 & - & 0.2 & 1.6 & - \\
T1a & 0.4 & 1.4 & 90 & 0.1 & & 0.3 & 20 \\
\hline
\end{tabular}

be understood in two alternative ways: first, T1a can be in an early stage and $\mathrm{CO}$ and $\mathrm{HCO}^{+}$have not yet had time to reach the steady state abundances. This alternative would then imply that $\mathrm{NH}_{3}$ and $\mathrm{HNC}$ are here early time species, and according to the models of Rawlings et al. (2002) would indicate an initially "H-rich" environment. The second alternative, and in fact the more likely, is that $\mathrm{CO}$ and $\mathrm{HCO}^{+}$are depleted in T1a. Previous observations have namely shown that $\mathrm{NH}_{3}$ can remain in the gas-phase in the situation where $\mathrm{CO}$ is heavily depleted (e.g., Willacy et al. 1998; Tafalla et al. 2002). These studies concern mainly starless cores, but may be valid for cores with low-mass protostars which have not affected their surroundings yet. The high fractional $\mathrm{HNC}$ and $\mathrm{HCN}$ abundances in T1a suggest furthermore that also these molecules are more resistant against freezing-out than $\mathrm{CO}$ and $\mathrm{HCO}^{+}$. This may be attributed to the replenishment of the $\mathrm{HNCH}^{+}$ion via the reaction between $\mathrm{NH}_{3}$ and $\mathrm{C}^{+}$. For the rest of the cores, Table 5 shows no definite trends which allow age sequencing. At the very best, our analysis indicates a possibility to discriminate cores with dynamical ages $\leq 10^{5}$ years from those older than $10^{6}$ years. However, to fully establish such a conclusion, a considerably larger sample of cores is needed.

\section{Summary}

We have completed a survey of dense cores in L 1251 in several high gas density tracers: $\mathrm{HCN}, \mathrm{HNC}, \mathrm{CS}$ and $\mathrm{HCO}^{+}$. On the larger scales, all observed molecules have similar distributions, including that of the previously published $\mathrm{NH}_{3}$ data. Velocity components observed are consistent with the ${ }^{13} \mathrm{CO}$ data by Sato et al. (1994). The "head" part of the cloud consists of two gas components, whose central velocities differ by about $1 \mathrm{~km} \mathrm{~s}^{-1}$. Altogether 15 dense cores can be identified in our maps.

Around IRAS $22343+7501$, which is proposed to power the extended $\mathrm{CO}$ outflow, we have detected $\mathrm{HCO}^{+}$wing emission, the distribution of which is resembling either a rotating disk or a dense outflow. Stability considerations seem to exclude a disk interpretation. If an outflow, the derived dynamical age and apparent orientations suggest that its origin is most likely different from that of the $\mathrm{CO}$ outflow. In the direction of IRAS 22343+7501 Beltrán et al. (2001) have detected two continuum sources, separated by $7^{\prime \prime}$, having spectral indices consistent with thermal emission. Thus, both sources could be protostars each driving its own outflow.
We have made additional observations towards five cores in $\mathrm{SO}, \mathrm{CH}_{3} \mathrm{CCH}$ and rare isotopomers of the mapped molecules in the selection. Using methyl acetylene and the $\mathrm{HCN} / \mathrm{HNC}$ ratios as thermometers, we find a "temperature gradient" in the cloud. The highest temperature is detected in the head region.

The derived column density ratios do not change much from core to core. This is probably traceable to the fact that all cores are star forming and most of the molecules observed are characteristic of mature chemistry. Two of the cores have, however, peculiar abundance ratios. The core located in the tip of the "head" of the cloud, has clearly higher $\mathrm{SO} / \mathrm{CS}$ and $\mathrm{NH}_{3} / \mathrm{CS}$ ratios than seen anywhere else in the cloud, suggesting that the core has reached a very late stage of chemical evolution, probably assisted by an elevated temperature due to shock - heating in the head. In the other exceptional core, lying in the more quiescent "tail" of the cloud, the column densities of $\mathrm{CO}$ and $\mathrm{HCO}^{+}$are low compared with those of $\mathrm{HNC}, \mathrm{HCN}$, and $\mathrm{NH}_{3}$. We suggest that this is due to depletion of $\mathrm{CO}$ and $\mathrm{HCO}^{+}$, and as a corollary, that also $\mathrm{HNC}$ and $\mathrm{HCN}$, like $\mathrm{NH}_{3}$ belong to the molecules that remain longer in the gas phase than $\mathrm{CO}$ and $\mathrm{HCO}^{+}$. The similar behavior of the former three molecules can probably be explained by their close relationship in the ionmolecule reaction schemes.

Four out of the five cores considered in L 1251 have embedded protostars in different stages of evolution. The revised average SFE of $\sim 10 \%$ is almost 3 times lower than the previous estimate, but still $\sim 5$ times higher than the overall SFE of the Galaxy. This high SFE indicates a contribution from externally triggered star formation.

Acknowledgements. The research was partly funded by the Ministry of Science and Technology of Serbia grant No. P1191 (2001-2004) and by the Finnish Center for International Mobility (CIMO). We are very grateful to Dr. M. Kun for the suggestion to use the 2MASS ASC, and to Dr. N. Mizuno who provided the ${ }^{13} \mathrm{CO}$ data presented in Fig. 1.

Onsala Space Observatory is the Swedish National Facility for Radio Astronomy and is operated by Chalmers University of Technology, Göteborg, Sweden, with financial support from the Swedish Natural Science Research Council and the Swedish Board for Technical Development.

\section{References}

Anderson, I. M., Caselli, P., Haikala, L. K., \& Harju, J. 1999, A\&A, 347,983

Askne, J., Höglund, B., Hjalmarson, Å., \& Irvine, W. M. 1984, A\&A, 130,311

Balázs, L. G., Eisloeffel, J., Holl, A., Kelemen, J., \& Kun, M. 1992, A\&A, 255281

Beltrán, M. T., Estalella, R., Anglada, G., Rodríguez, L. F., \& Torrelles, J. M. 2001, AJ, 121, 1556

Berkhuijsen, E. M. 1971, A\&A, 14, 252

Casanova, S., Montmerle, T., Feigelson, E. D., \& André, P. 1995, ApJ, 439, 752

Caselli, P., Benson, P. J., Myers, P. C., \& Tafalla, M. 2002, ApJ, 572, 238

Claussen, M. J., Wilking, B. A., Benson, P. J., et al. 1996, ApJS, 106, 111

Dickman, R. L., \& Clemens, D. P. 1983, ApJ, 271, 143

Eiroa, C., Torrelles, J. M., Miranda, L. F., Anglada, G., \& Estalella, R. 1994, A\&AS 108, 73 
Frerking, M. A., Langer, W. D., \& Wilson, R. W. 1982, ApJ, 262, 590 Fridlund, C. V. M., Bergman, P., White, G. J., Pilbratt, G. L., \& Tauber, J. A. 2002, A\&A, 382, 573

Getman, K. V., Fiegelson, E. D., Townsley, L., et al. 2002, ApJ, 575, 354

Grenier, I. A., Lebrun, F., Arnaud, M., et al. 1989, ApJ, 347, 231

Grissom Meehan, L. S., Wilking, B. A., Claussen, M. J., Mundy, L. G., \& Wootten, A. 1998, AJ, 115, 1599

Herbst, E., \& Leung, C. M. 1989, ApJS, 69, 271

Hirota, T., Yamamoto, S., Mikami, H., \& Ohisi, M. 1998, ApJ, 503, 717

Johansson, L. E. B., Greve, A., Booth, R. S., et al. 1998, A\&A, 331, 857

Kontinen, S., Harju, J., Heikkilä, A., \& Haikala, L. K. 2000, A\&A, 361,704

Kun, M. 1982, Afz, 18, 63

Kun, M. 1998, ApJS, 115, 59

Kun, M., \& Prusti, T. 1993, A\&A, 272, 235

Kun, M., Vinkó, J., \& Szabados, L. 2000, MNRAS, 319, 777

Lepp, S., \& McCray, R. 1983, ApJ, 269, 560

Lynds, B. T. 1962, ApJS, 7, 1

Mardones, D., Myers, P. C., Tafalla, M., et al. 1997, ApJ, 489, 719

Myers, P. C., Dame, T. M., Thaddeus, P., et al. 1986, ApJ, 301, 398

Nejad, L. A. M., Williams, D. A., \& Charnley, S. B. 1990, MNRAS, 246, 183
Nejad, L. A. M., \& Wagenblast, R. 1999, A\&A, 350, 204

Nikolić S., Kiss Cs., Johansson, L. E. B., Wouterloot J. G A., \& Tóth L. V. 2001, A\&A, 367, 694

Nilsson, A., Hjalmarson, A., Bergamn, P., \& Millar, T. 2000, A\&A 358, 257

Rawlings, J. M. C., Hartquist, T. W., Williams, D. A., \& Falle, A. E. G. 2002, A\&A, 391, 681

Rosvick, J. M., \& Davidge, T. J. 1995, PASP, 107, 49

Sato, F., Mizuno, A., Nagahama, T., et al. 1994, ApJ, 435, 279

Sato, F., \& Fukui, Y. 1989, ApJ, 343, 773

Schwartz, P. R., Gee G., \& Huang, Y.-L. 1988, ApJ, 327, 350

Smith, M. D., \& Rosen, A. 2003, MNRAS, 339, 133

Tafalla, M., Myers, P. C., Caselli, P., Walmsley, C. M., \& Comito, C. 2002, ApJ, 569, 815

Torrelles, J. M., Rodríguez, L. F., Cantó, J., et al. 1983, ApJ, 274, 214

Tóth, L. V., \& Walmsley, C. M. 1994, IBVS, 4107

Tóth, L. V., \& Walmsley, C. M. 1996, A\&A, 311, 981

Tóth, L. V., \& Kun, M. 1997, IBVS, 4492

Wilking, B. A., Claussen, M. J., Benson, P. J., et al. 1994, ApJ, 431, L119

Willacy, K., Langer, W. D., \& Velusamy, T. 1998, ApJ, 507, 171

Wilson, T. L., \& Rood, R. 1994, ARA\&A, 32, 191

Xiang, D., \& Turner, B. T. 1995, ApJS, 99, 121

Yun, J. L., Moreira, M. C., Afonso, J. M., \& Clemens, D. P. 1999, AJ, 118,990 